\title{
Phase-field Modeling of Precipitate Behavior in RPV Steel Using CALPHAD Database
}

\author{
Kunok Chang ${ }^{1,2}, *$, Junhyun Kwon ${ }^{1}$, and Gyeong-Geun Lee ${ }^{1}$ \\ ${ }^{1}$ Korea Atomic Energy Research Institute, Daejeon 34057, Republic of Korea \\ ${ }^{2}$ Nuclear Engineering Department, Kyung Hee University, Yongin 17104, Republic of Korea
}

\begin{abstract}
We developed a multi-scale modeling framework using the thermodynamic UW1 database of the Fe-Mn-Ni-Si quaternary system. To obtain input data for the phase-field model, such as mobility and interfacial energy data, we used DICTRA software and the TC-PRISMA package with the commercial TCFE8 and MOBFE3 databases. Using the developed framework, we evaluated the stability of single precipitates and compared the results to those from the thermodynamic model. We also investigated the precipitate interactions of paired particles of identical and different phases.
\end{abstract}

(Received February 5, 2018; Accepted April 18, 2018)

Keywords: particle stability, particle interaction, phase-field modeling

\section{INTRODUCTION}

Predicting multi-particle behavior is an extremely complicated task. The Ostwald ripening of single-phase precipitates has been extensively investigated using both analytical [1-8] and numerical methods [8-15]. The role of the coarsened phase volume fraction has been explored $[3,5,6,9,10]$ and morphological deviations from the spherical (circular) coarsened phases at high volume fractions have already been observed in previous studies $[6,9,10,15]$. Ardell evaluated the role of the coarsened phase fraction using a theoretical approach and he found that the peak of the particle size distribution decreased as the particle fraction increased [3]. Brasilsford and Wynblatt [7] also found that the particle size distribution peak position decreased as the volume fraction increased, and they claimed that the effect was much less sensitive to volume fraction than proposed by Ardell [3]. Although the microstructural evolutions driven by curvature minimization have been studied extensively for many decades, current understanding of Ostwald ripening remains limited. Because we have no analytical solutions for the diffusion equations in most cases and ripening is

*Corresponding Author: Kunok Chang

[Tel: +82-31-201-3683, E-mail: kunok.chang@khu.ac.kr]

Copyright (c) The Korean Institute of Metals and Materials extremely complex, multi-particle behaviors are usually predicted based on the mean-field assumption [1-8]. or numerical approaches [6,8-10,15]. Previous simulations of particle coarsening have predicted the behaviors of single phases using phenomenological models $[9,10,15]$. In this study, we performed a simulation of multiphase precipitates using a CALPHAD-type database to predict the behaviors of realistic precipitates. The major elements of low- $\mathrm{Cu}$ reactor pressure vessel (RPV) steel are $\mathrm{Fe}, \mathrm{Mn}, \mathrm{Ni}$ and $\mathrm{Si}$ [16-18]; therefore, we selected the $\mathrm{Fe}-\mathrm{Mn}-\mathrm{Ni}-\mathrm{Si}$ (hereafter FMNS) quaternary system to investigate multi-phase coarsening, using the FMNS database. The phase-field method has been extensively applied to investigate the microstructural evolution of low-alloy Fe-based steel [19-21] assuming only one precipitate phase within the system. T3, T6, and $\mathrm{T} 7$ $\mathrm{Mn}-\mathrm{Ni}-\mathrm{Si}$-rich (MNS) precipitates are generally observed in RPV steel [16]; therefore, we assumed the presence of three types of MNS precipitates in the system. We adopted the Kim-Kim-Suzuki (KKS) model [22] to perform multi-phase and multi-component phasefield modeling. The FMNS system, a prototype of RPV steel, is of great interest in nuclear engineering applications [16-18,23,24]. Because MNS precipitates are among the main sources of late-stage hardening for FMNS [16-18,23,24], we examined the stability of the MNS precipitates. The $\mathrm{T} 3, \mathrm{~T} 6$, and $\mathrm{T} 7$ precipitate 
phases, major types of MNS precipitates, were considered in the UW1 database [16] in this study; we also simulated the evolution of pairs of T3-T3, T7-T7, and $\mathrm{T} 3-\mathrm{T} 7$ precipitates to quantify the interactions between particles.

\section{UW1 CALPHAD DATABASE}

In the UW1 database [16], one bcc phase representing the matrix and 12 MNS precipitate phases are considered. In our study, we selected the matrix phase and three MNS precipitate phases for simplicity. The thermodynamic parameters we used were taken from the supplementary material of ref. [16] as follows:

for bcc FMNS phase,

$$
\begin{aligned}
& { }^{0} L_{F e, M n}^{b c c}=-2759+1.23 T \\
& { }^{0} L_{F e, N i}^{b c c}=-956.63-1.28726 T \\
& { }^{1} L_{F e, N i}^{b c c}=1789.03-1.92912 T \\
& { }^{0} L_{F e, S i}^{b c c}=-153138.56+46.86 T \\
& { }^{1} L_{F e, S i}^{b c c}=-92352,{ }^{2} L_{F e, S i}^{b c c}=62240 \\
& { }^{0} L_{M n, N i}^{b c}=-3508.43-23.7885 T \\
& { }^{0} L_{M n, S i}^{b c c}=-89620.7+2.9410 T \\
& { }^{1} L_{M n, S i}^{b c c}=-7500.0 \\
& { }^{0} T_{F e, M n}^{b c c}=123.0,{ }^{0} T_{F e, S i}^{b c c}=504.0
\end{aligned}
$$

for the T3: $M n_{6 / 29} N i_{16 / 29} S i_{7 / 29}$ phase,

$$
\begin{aligned}
{ }^{0} g_{M n, N i, S i}^{T 3}= & -48186.497+6 / 29^{0} G_{M n}^{c b c c}+16 / 29^{0} G_{N i}^{f c c} \\
& +7 / 29^{0} G_{S i}^{\text {diamond }}
\end{aligned}
$$

for the T6: $\mathrm{Mn}_{1 / 3}(\mathrm{Ni}, \mathrm{Si})_{2 / 3}$ phase,

$$
\begin{aligned}
& { }^{0} g_{M n, N i}^{T 6}=10086.99+1 / 3^{0} G_{M n}^{c b c c}+2 / 3^{0} G_{N i}^{f c c} \\
& { }^{0} g_{M n, S i}^{T 6}=1666.67+1 / 3^{0} G_{M n}^{c b c c}+2 / 3^{0} G_{S i}^{f c c} \\
& { }^{0} L_{M n, N i, S i}^{b c c}=-159474.81,{ }^{1} L_{M n, N i, S i}^{b c c}=-172110.47
\end{aligned}
$$

and for the T7: $\mathrm{Mn}_{1 / 2} \mathrm{Ni}_{1 / 3} S i_{1 / 6}$ phase,

$$
\begin{aligned}
{ }^{0} g_{M n, N i, S i}^{T 7}= & -32434.25-5 T+1 / 2^{0} g_{M n}^{c b c c}+1 / 3^{0} g_{N i}^{f c c} \\
& +1 / 6^{0} g_{S i}^{\text {diamond }}
\end{aligned}
$$

\section{PHASE-FIELD MODEL AND INPUT PARAMETER EVALUATIONS}

We utilize the phase-field model to simulate the microstructural evolution of the FMNS system by solving the Cahn-Hilliard [25] and Allen-Cahn (GinzburgLandau) equations [26] to simulate the microstructural evolution. We will denote the composition $(i=1,2$, 3 , and 4 for $\mathrm{Fe}, \mathrm{Mn}, \mathrm{Ni}$, and $\mathrm{Si}$, respectively) in phase $\theta$ using $c_{i}^{\theta}(r, t)$ at position $r$ and time $t . \theta$ indicates the $\mathrm{T} 3, \mathrm{~T} 6$, and $\mathrm{T} 7$ phases. We introduce four non-conserved order parameters $\left(\phi_{i}\right)$ to indicate the regions of the four precipitated phases. The composition $c_{i}(r, t)$ is given as follows [22]:

$$
\begin{aligned}
c_{i}(r, t) & =c_{i}^{T 3}(r, t) h\left(\phi^{T 3}\right)+c_{i}^{T 6}(r, t) h\left(\phi^{T 6}\right)+c_{i}^{T 7}(r, t) h\left(\phi^{T 7}\right) \\
& +c_{i}^{\alpha}\left[1-\Sigma_{\theta} h\left(\phi^{\theta}\right) .\right.
\end{aligned}
$$

where [27],

$$
h\left(\phi^{\theta}\right)=\sum_{i=1}^{n p}\left(\phi_{i}^{\theta}\right)^{3}\left[6\left(\phi_{i}^{\theta}\right)^{2}-15 \phi_{i}^{\theta}+10\right]
$$

where $n p$ represents the number of $\theta$ precipitate.

The local free energy density $G\left(c_{i}^{\theta}, t\right)$ of the system is expressed as follows:

$$
\begin{aligned}
G\left(c_{i}^{\theta}, t\right) & =\int \frac{\rho}{M}\left[h\left(\phi^{T 3}\right) g^{T 3}+h\left(\phi^{T 6}\right) g^{T 6}+h\left(\phi^{T 7}\right) g^{T 7}\right. \\
& \left.+\left[1-\Sigma_{\theta} h\left(\phi^{\theta}\right)\right] g^{\alpha}\left(c_{i}^{\alpha}, t\right)+g\left(\phi_{i}^{\theta}\right)\right] d V
\end{aligned}
$$

or

$$
\begin{aligned}
g\left(c_{i}^{\theta}, t\right) & =h\left(\phi^{T 3}\right) g^{T 3}+h\left(\phi^{T 6}\right) g^{T 6}+h\left(\phi^{T 7}\right) g^{T 7} \\
& +\left[1-\Sigma_{\theta} h\left(\phi^{\theta}\right)\right] g^{\alpha}\left(c_{i}^{\alpha}, t\right)+g\left(\phi_{i}^{\theta}\right)
\end{aligned}
$$

where the units of all free energy densities (expressed by $g$ in this study) in this study are joules per mole: the unit of total free energy of the system $G$ is $J$. We assumed that density $\rho$ is $7.9 \mathrm{~g} / \mathrm{cm}^{3}$ and the atomic molar mass $M$ is 56 .

$$
\begin{aligned}
& g^{\alpha}\left(c_{i}^{\alpha}, t\right)=c_{1}^{\alpha} \times{ }^{0} g_{F e}^{b c c}+c_{2}^{\alpha} \times{ }^{0} g_{M n}^{b c c}+c_{3}^{\alpha} \times{ }^{0} g_{N i}^{b c c}+c_{4}^{\alpha} \times{ }^{0} g_{S i}^{b c c} \\
& +R T\left[c_{1}^{\alpha} \log c_{1}^{\alpha}+c_{2}^{\alpha} \log c_{2}^{\alpha}+c_{3}^{\alpha} \log c_{3}^{\alpha}+c_{4}^{\alpha} \log c_{4}^{\alpha}\right]+L_{F e, M n}^{b c c} c_{1}^{\alpha} c_{2}^{\alpha} \\
& +L_{F e, N i}^{b c c} c_{1}^{\alpha} c_{3}^{\alpha}+L_{F e, S i}^{b c c} c_{1}^{\alpha} c_{4}^{\alpha}+L_{F e, N i}^{b c c} c_{2}^{\alpha} c_{3}^{\alpha}+L_{M n, S i}^{b c c} c_{2}^{\alpha} c_{4}^{\alpha}
\end{aligned}
$$

And 


$$
g\left(\phi_{i}^{\theta}\right)=\frac{\omega_{p}}{2}\left(\phi_{i}^{\theta}\right)^{2}\left(\phi_{i}^{\theta}-1\right)^{2}+\gamma\left(\phi_{i}^{\theta}\right)^{2} \Sigma_{j \neq i, \theta}\left(\phi_{i}^{\theta}\right)^{2}
$$

with $c_{1}^{\alpha}=1.0-c_{2}^{\alpha}-c_{3}^{\alpha}-c_{4}^{\alpha}$. We chose $\omega_{p}=200.0$ and $\gamma=5.0$ in Eq. 5. From ref. [28], we obtain the free energy of each element of the $\alpha$ phase. The free energies of the T3, T6, and T6 precipitates is given as follows:

$$
\begin{aligned}
& g^{T 3}={ }^{0} g_{M n, N i, S i}^{T 3} \\
& g^{T 6}\left(c_{i}^{T 6}, t\right)=y_{3}^{I I} \times{ }^{0} g_{M n, N i}^{T 6}+y_{4}^{I I} \times{ }^{0} g_{M n, S i}^{T 6} \\
& +\frac{2}{3} R T\left[y_{3}^{I I} \log y_{3}^{I I}+y_{4}^{I I} \log y_{4}^{I I}\right]+y_{3}^{I I} \log y_{4}^{I I} L_{M n, N i, S i}^{b c c}
\end{aligned}
$$

where $y_{3}^{I I}$ and $y_{4}^{I I}$ denote the site fraction of $\mathrm{Ni}$ and $\mathrm{Si}$ at the second sub-lattice of the $\mathrm{T} 6$ phase, respectively.

$$
g^{T 7}={ }^{0} g_{M n, N i, S i}^{T 7}
$$

We solve the Ginzburg-Landau equation in Eq. 9 and the Cahn-Hilliard equation in Eq. 10.

$$
\begin{aligned}
& \frac{\partial \phi_{i}^{\theta}(r, t)}{\partial t}=-L^{\theta} \frac{\delta_{g}}{\delta \phi_{i}^{\theta}(r, t)}(\theta=\mathrm{T} 3, \mathrm{~T} 6, \text { and T7) } \\
& \frac{\partial c_{I}(r, t)}{\partial t}=\nabla\left(D_{i}\left(\phi^{\theta}\right) \nabla c_{i}\right)+\nabla\left(D_{i}\left(\phi^{\theta}\right) h^{\prime}\left(\phi^{\theta}\right)\left(c_{i}^{\alpha}-c_{i}^{\theta}\right) \nabla \phi^{\theta}\right)
\end{aligned}
$$

We implemented the forward Euler scheme to solve Eq. 9 and 10. To determine $D_{i}$ in Eq. 10 we adopted the relation in refs. [20,21].

$$
D_{i}\left(\phi^{\theta}, T\right)=\left(1-\Sigma_{j} \phi_{j}^{\theta}\right) D_{i}^{\alpha}(T)+\Sigma_{j} \phi_{j}^{\theta} D_{i}^{\theta}(T)
$$

We use the relation $D_{i}^{\theta}(T)={ }^{0} D_{i}^{\theta} \exp \left(-\frac{Q_{i}^{\theta}}{R T}\right)$ to determine the diffusivity; the parameters used to determine this value are listed in Table 1.

In principle, one has to know the diffusivity data of each element of each precipitated phase to perform phase-field modeling. However, the diffusivity data of T3, T6, and T7 phases are not available. Since a precipitate larger than $17 \mathrm{~nm}$ has the FCC crystal structure [30], we adopted the diffusion data of each element in the Austenite (FCC) phase for all precipitated phases.

We could not find diffusivity data for $\mathrm{Si}$ in the $\gamma($ fcc $)$ phase; therefore, we calculate it from a kinetic simulation. We performed a DICTRA simulation using Thermo-Calc 2017a software with the TCFE 8 and MOBFE3 databases. At $\mathrm{T}=550 \mathrm{~K}$, in the $\mathrm{Fe}-\mathrm{Si}$ binary system ( $\gamma(\mathrm{fcc})$ phase) the $\mathrm{Si}$ mole fraction is always 0.03 at $\mathrm{x}=0$; the value is 0.005 initially, except for the point of $\mathrm{x}=0$ in Fig. 1. From the diffusion length depending on the simulation time, we calculated the diffusion coefficient of Si in the $\gamma(\mathrm{fcc})$ phase.

We restated Eq. 9 as Eq. 12.

$$
\frac{\partial \phi_{i}^{\theta}(r, t)}{\partial t}=-L^{\theta}\left(\frac{\partial G}{\partial \phi_{i}^{\theta}(r, t)}-\omega^{\theta} \nabla^{2} \phi_{i}^{\theta}\right)
$$

$(\theta=\mathrm{T} 3, \mathrm{~T} 6$, and $\mathrm{T} 7)$

In our simulations, we use the energy normalized by $R T$, where $R=8.3144598 \mathrm{~J} / \mathrm{mol} \cdot \mathrm{K}$ and the temperature $\mathrm{T}=550 \mathrm{~K}$. The diffusivity values are normalized by $D_{2}^{\alpha}(T)$. For the length of the grid spacing, $\Delta x=\Delta y$ was taken as $0.125 \mathrm{~nm}$ [21]. The time in our study is normalized by $0.01 \mathrm{~s}$.

To determine $\omega^{\theta}$ in Eq. 12, the interfacial energies between the matrix and precipitate phases are needed. So far, we have not found any literature that has evaluated the interfacial energies relevant for our study; therefore, we measure the interfacial energy using the extended Becker's model function [31]. To perform the interfacial energy estimation, we used TC-PRISMA software (included in Thermo-Calc 2016a) with the TCAL3 and MOBAL3 databases. However, evaluating the interfacial energy for the whole $\mathrm{Si}$ fraction in the T6 phase using TC-PRISMA is not possible. We find that the $\mathrm{Si}$ fraction in the $\mathrm{T} 6$ phase is generally smaller than 0.3 ; therefore, we assume that the interfacial energy is given by Eq. 13. $0.2496 \mathrm{~J} / \mathrm{m}^{2}$ [22].

Table 1. The values of the maximal diffusion coefficient and activation energy of each element [29].

\begin{tabular}{cccc}
\hline Elements & Phase & ${ }^{0} D_{i}^{\theta}\left(\mathrm{m}^{2} / \mathrm{s}\right)$ & $Q_{i}^{\theta}(\mathrm{J} / \mathrm{mol})$ \\
\hline $\mathrm{Mn}$ & $\alpha(\mathrm{bcc})$ & $1.5 \times 10^{-4}$ & $2.34 \times 10^{5}$ \\
\hline $\mathrm{Mn}$ & $\gamma(\mathrm{fcc})$ & $1.6 \times 10^{-5}$ & $2.62 \times 10^{5}$ \\
\hline $\mathrm{Ni}$ & $\alpha(\mathrm{bcc})$ & $4.2 \times 10^{-3}$ & $2.68 \times 10^{5}$ \\
\hline $\mathrm{Ni}$ & $\gamma(\mathrm{fcc})$ & $7.7 \times 10^{-5}$ & $2.81 \times 10^{5}$ \\
\hline $\mathrm{Si}$ & $\alpha(\mathrm{bcc})$ & $1.7 \times 10^{-4}$ & $2.29 \times 10^{5}$ \\
\hline
\end{tabular}


For the T3 and T7 stoichiometric compounds, we also evaluate the interfacial energies using TC-PRISMA as 0.3311 and $0.1791 \mathrm{~J} / \mathrm{m}^{2}$. The interfacial energy is given by Eq. 13 when the gradient coefficient is $\varepsilon^{2}$.

$$
E=\frac{\varepsilon \sqrt{\varepsilon^{\theta}}}{3 \sqrt{2}}
$$

Since we used the normalized variables in this study, we evaluated the normalized interfacial energy.

$$
E^{*}=\frac{\varepsilon^{*, \theta} \sqrt{\omega^{*, \theta}}}{3 \sqrt{2}}
$$

The unit of $\varepsilon$ is $\mathrm{J} / \mathrm{m}^{2} \cdot \mathrm{mol}$. We chose $\varepsilon^{*, T 6}=1.0$ as a non-dimensional parameter which corresponds to the interfacial energy $0.26 \mathrm{~J} / \mathrm{m}^{2}$ which is quite comparable to the value $0.2496 \mathrm{~J} / \mathrm{m}^{2}$ which we obtained from the TC-Prisma calculation. We also determined $\varepsilon^{*, T 3}=1.33$ and $\varepsilon^{*, T 7}=0.72$ using a consistent way.

\section{SIMULATION DETAILS}

We performed the simulations with a simulation cell size of $8 \times 8 \mathrm{~nm}^{2}$. The initial precipitate radius was $1.5 \mathrm{~nm}$. The discretized time step was selected as given in Table 2.

We chose $\Delta t$ after convergence and accuracy testing. The simulations obtained results in 1 to $12 \mathrm{~h}$, depending on the $\Delta t$ of the system. For the matrix, the initial compositions in the $\alpha$ phase were $c_{2}^{\alpha}=0.008$, $c_{3}^{\alpha}=0.008$, and $c^{\alpha}=0.00808$. For the T6 precipitate,

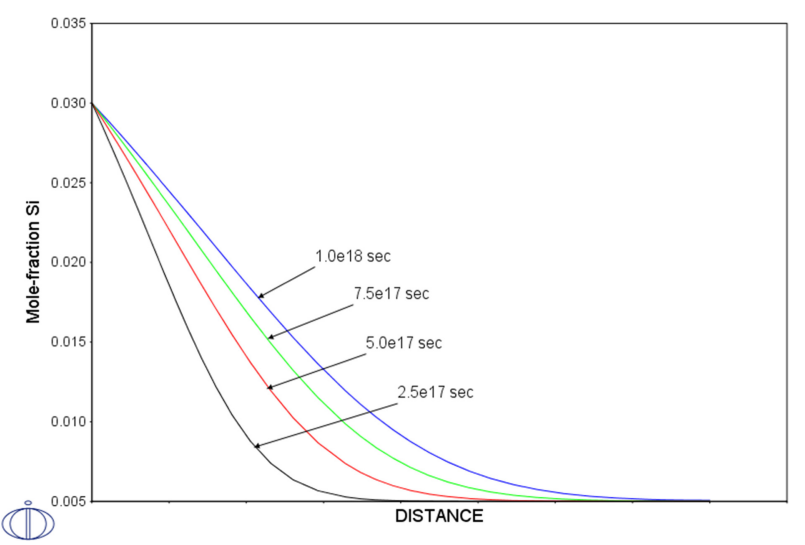

Fig. 1. Profile of Si mole-fraction at four different times.
Table 2. Normalized $\Delta t^{*}$ for six precipitate configurations

\begin{tabular}{cc}
\hline Precipitate & $\Delta t^{*}$ \\
\hline Single T3 & $1.0 \times 10^{-4}$ \\
\hline Single T6 & $1.0 \times 10^{-4}$ \\
\hline Single T7 & $1.0 \times 10^{-4}$ \\
\hline T3-T3 & $1.0 \times 10^{-4}$ \\
\hline T7-T7 & $1.0 \times 10^{-4}$ \\
\hline T3-T7 & $1.0 \times 10^{-5}$ \\
\hline
\end{tabular}

$c_{3}^{T 6}=0.459234067$ and $c_{4}^{T 6}=0.2074326$.

For the $\mathrm{T} 3$ and $\mathrm{T} 7$ cases, the compositions of the precipitates were fixed and the initial compositions of the $\alpha$ phase were assumed to be equal to those of the T6 case. For the case with a single precipitate, we placed a circular precipitate with an initial radius $r=1.5 \mathrm{~nm}$ at the center of the simulation cell. For multiple precipitate phases, we placed two precipitates with initial radii $r=1.5 \mathrm{~nm}$ at two symmetrical positions with the distances $L=3.25,3.50$, and $3.75 \mathrm{~nm}$ relative to the center of the system.

\section{RESULTS}

We performed the simulation to examine the stability of a single precipitate as shown in Fig. 2. In Fig. 3(a), the T6 precipitate undergoes minor accommodation at a very early stage. After $0.0002 \mathrm{~s}$, the precipitate radii remains the same as it was during the modeling in Fig. 3(b). This means that the T6 precipitate is thermodynamically stable. Because the T3 and T7 phases are unstable, these precipitates shrink with time; the $\mathrm{T} 7$ precipitate radius decreases more rapidly than that of the $\mathrm{T} 3$ precipitate. We performed CALPHAD modeling using Thermocalc software and the UW1 database [16] with $c_{2}^{\alpha}=0.008, c_{3}^{\alpha}=0.008, c_{4}^{\alpha}=0.00808$, and $\mathrm{T}=550 \mathrm{~K}$. The equilibrium phases were $\operatorname{bcc}(\mathrm{A} 2)$ (98.940 $\mathrm{mol} \%)$ and T6 (1.363 mol\%). Therefore, we concluded that the phase-field simulation with a single precipitate was consistent with the thermodynamic model. As shown in Fig. 4, the diffusion field of the solutes overlapped between the particles, therefore, the solute concentration is relatively higher than in other 
matrix regions. As a result, the dissolution rate decreases along the direction of the other particle. As a result, the particle shape becomes asymmetric in Fig. 4.

Therefore, the T3 particles remain longer for two interacting T3 particles compared to a single T3 particle. In addition, when the distance between

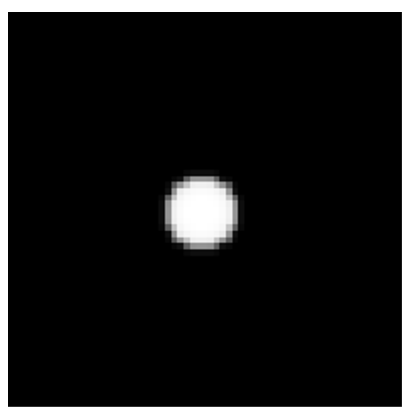

Fig. 2. $\phi^{T 3}$ profile when $t=6 \mathrm{~s}$. The figure is visualized by mapping a summation of the $\phi^{T 3}$ value to a gray scale (maximum 1.0 , minimum 0.0 ).
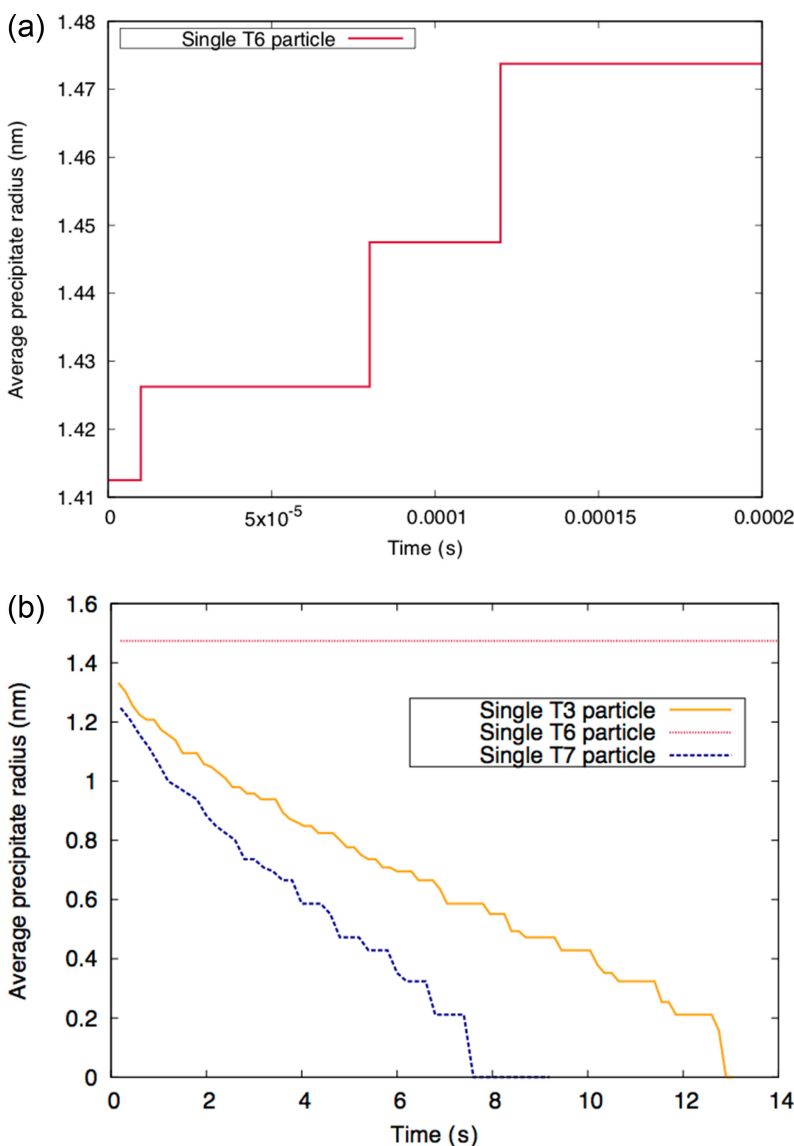

Fig. 3. (a) Evolution of radii of T6 precipitate at very early stage $(<0.0002 \mathrm{~s})$, (b) precipitate radii of T3, T6, and T7 phases with respect to time for the case of single-precipitate simulation. particles $L$ is $3.75 \mathrm{~nm}$, the particle size evolution curve shown in Fig. 5 approaches convergence with that curve of the single T3 particle. Consistent results

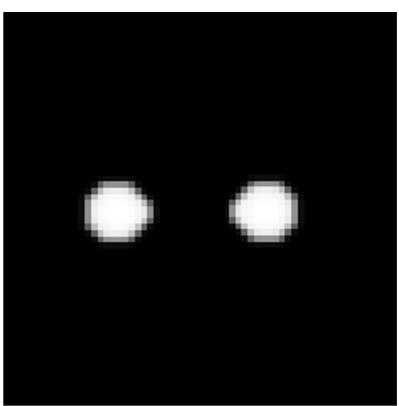

Fig. 4. $\phi_{1}^{T 3}$ and $\phi_{2}^{T 3}$ profiles when $t=6 \mathrm{~s}$. The distance between the particle centers $\mathrm{L}=3.25 \mathrm{~nm}$ when $t=6 \mathrm{~s}$. The figure was visualized by mapping a summation of the $\phi_{1}^{T 3}+\phi_{2}^{T 3}$ value to a gray scale (maximum 1.0, minimum 0.0).

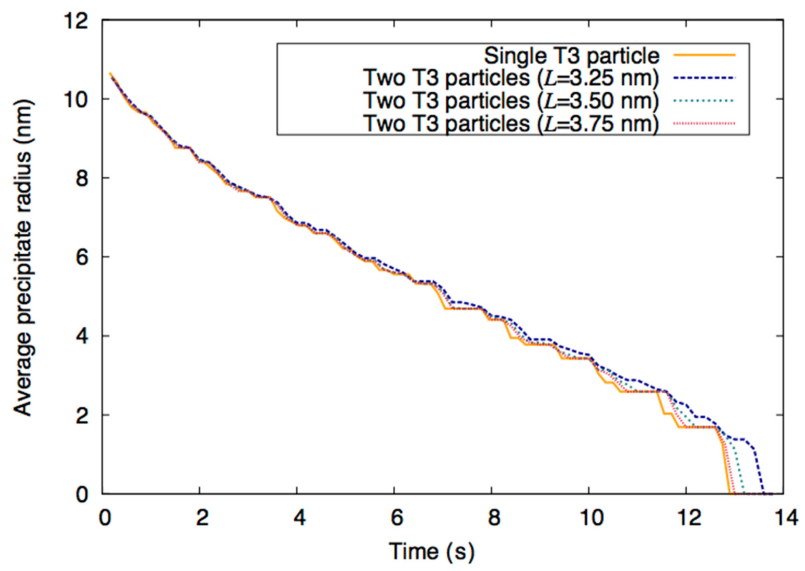

Fig. 5. Precipitate radius of $\mathrm{T} 3$ with respect to time for a single $\mathrm{T} 3$ precipitate versus two T3 precipitates separated by different distances $L$.

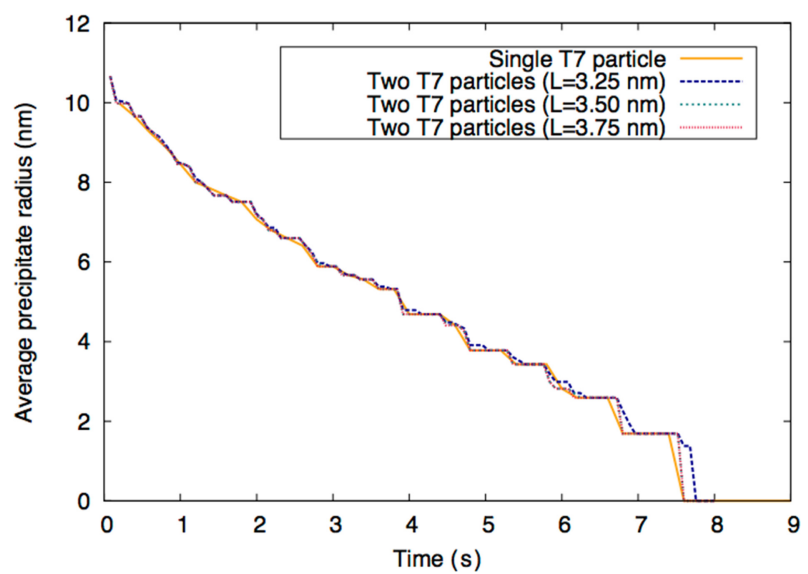

Fig. 6. Precipitate radius of T7 precipitate with respect to time for a single $\mathrm{T} 7$ precipitate and two $\mathrm{T} 7$ precipitates with varying distances between particles $L$. 


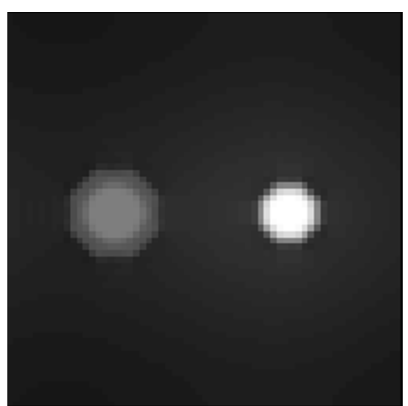

Fig. 7. Profile of Mn concentration when $t=1.5 \mathrm{~s}$. The distance between the particle centers $L$ is $3.25 \mathrm{~nm}$. The figure was visualized by mapping the $c_{2}$ value to a gray scale (maximum 0.5 , minimum $0.05)$.

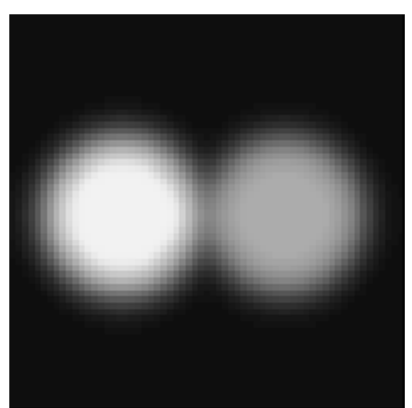

Fig. 8. Profile of $\mathrm{Ni}$ concentration when $t=1.5 \mathrm{~s}$. The distance between the particle centers $L$ is $3.25 \mathrm{~nm}$. The figure was visualized by mapping the $c_{3}$ value to a gray scale (maximum 0.6 , minimum $0.0)$.

are observed for the T7 precipitate in Fig. 6.

We performed a set of simulations to investigate the interactions between $\mathrm{T} 3$ and $\mathrm{T} 7$ precipitates, with $\mathrm{T} 3$ and T7 precipitates located as described in Fig. 7 and 8.

In Fig. 9 and 10, we find that the $\mathrm{T} 3$ and $\mathrm{T} 7$ precipitates survive longer under $\mathrm{T} 3-\mathrm{T} 7$ interactions. Even though the precipitates are of different phases, particle-particle interactions increase the lifetime of the precipitates, i.e., enhance their stability.

\section{CONCLUSIONS}

We developed a multi-scale modeling framework to simulate the microstructural evolution of MNS precipitates in the quaternary FMNS system representing RPV steel. The UW1 quaternary database was implemented and the DICTRA and TC-PRISMA packages were used to evaluate the mobility of solutes and the interfacial energies between the matrix phase

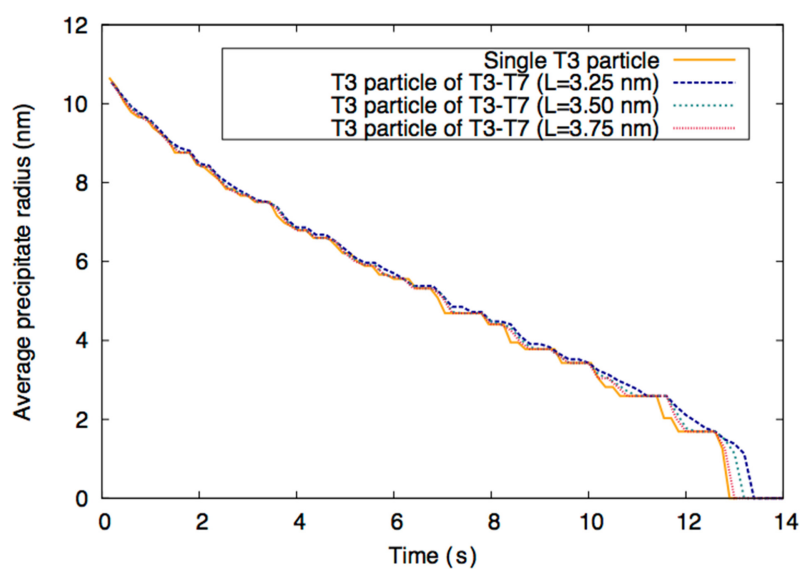

Fig. 9. Precipitate radius of $\mathrm{T} 3$ for a single $\mathrm{T} 3$ precipitate and paired T3-T7 precipitates with varying distances between particles $L$.

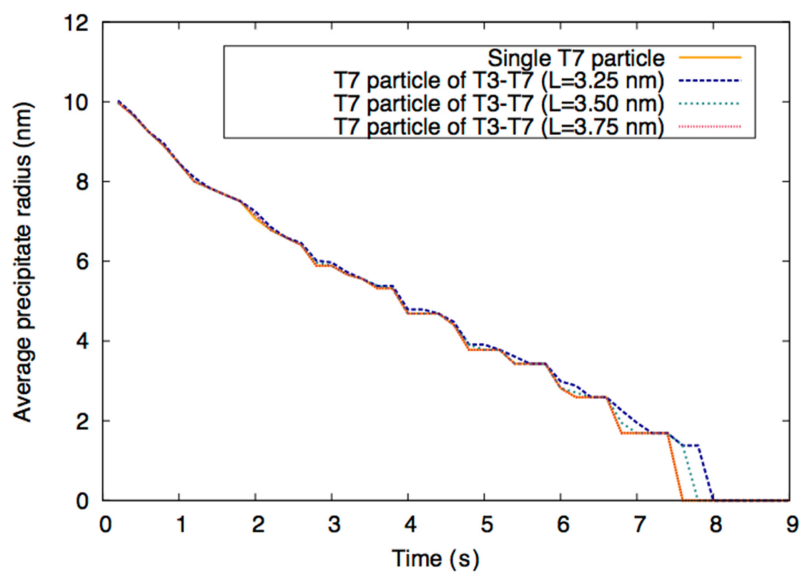

Fig. 10. Precipitate radius of $T 7$ for a single $T 7$ precipitate and paired $\mathrm{T} 3-\mathrm{T} 7$ precipitates with varying distances between particles $L$.

and precipitate phases. The results of the singleprecipitate simulations were consistent with the thermodynamic model. We also evaluated the interactions between precipitates and found that the precipitates survived longer when two particles were present, whether of the same or different phases, compared to the particle lifetime in the single-precipitate case.

\section{AKNOWLEDGEMENT}

This work was supported by a National Research Foundation of Korea(NRF) grant funded by the government of Korea (Ministry of Science and ICT) (NRF-2017M2A8A4015157). 


\section{REFERENCES}

1. I. M. Lifshitz and V. V. lyozov, J. Phys. Chem. Solids 19, 35 (1961).

2. Garay-Reyes C G, S. E. Hernández-Martínez, J. L. Hernández-Rivera, J. L. Hernández-Rivera, E. J. GutiérrezCastañeda, H. J. Dorantes-Rosales, J. Aguilar-Santillan, R. Martínez-Sánchez, Met. Mater. Int. 23, 298 (2017).

3. A. Ardell, Acta Metall. 20, 61 (1972).

4. P. W. Voorhees, J. Stat. Phys. 38, 231 (1985).

5. C. Davies, P. Nash, and R. N. Stevens, Acta Metall. 28, 179 (1980).

6. P. Voorhees and M. Glicksman, Metall. Mater. Trans. A 15, 1081 (1984).

7. A. Brailsford and P. Wynblatt, Acta Metall. 27, 489 (1979).

8. J. H. Yao, K. Elder, H. Guo, and M. Grant, Phys. Rev. B 47, 14110 (1993).

9. D. Fan, S. Chen, L. Q. Chen, and P. W. Voorhees, Acta Mater. 50, 1895 (2002).

10. S. G. Kim, Acta Mater. 55, 6513 (2007).

11. J. A. Warren, B. T. Murray, Modelling Simul. Mater. Sci. Eng. 4, 215 (1996).

12. T. Kraska, J. Phys. Chem. B 112, 12408 (2008).

13. G. Pévot, Phys. Rev. B 84, 045434 (2011).

14. P. W. Voorhees and M. Glicksman, Acta Metall. 32, 2013 (1984).

15. P. W. Voorhees, G. McFadden, R. Boisvert, and D. Meiron,
Acta Metall. 36, 207 (1988).

16. W. Xiong, H. Ke, R. Krishnamurthy, P. Wells, L. Barnard, G. R. Odette, and D. Morgan, MRS Commun. 4, 101 (2014).

17. D. Sprouster, J. Sinsheimer, E. Dooryhee, S. Ghose, P. Wells, T. Stan, N. Almirall, G. Odette, L. Ecker, Scripta Mater. 113, 18 (2016).

18. S. Hong, C. Lee, M.-C. Kim, and B.-S. Lee, Korean J. Met. Mater. 55, 752 (2017).

19. T. Koyama and H. Onodera, Mater. Trans. 46, 1187 (2005).

20. T. Koyama, K. Hashimoto, and H. Onodera, Mater. Trans. 47, 2765 (2006).

21. S. Biner, W. Rao, Y. Zhang, J. Nucl. Mater. 468, 9 (2016).

22. S. G. Kim, W. T. Kim, and T. Suzuki, Phys. Rev. E 60, 7186 (1999).

23. G. R. Odette and G. Lucas, JOM 53, 18 (2001).

24. G. R. Odette and G. Lucas, Radiat Eff. Defects Solids 144, 189 (1998).

25. J. W. Cahn and J. E. Hilliard, J. Chem. Phys. 28, 258 (1958).

26. S. M. Allen and J. W. Cahn, Acta Metall. 27, 1085 (1979).

27. J. Zhu, T. Wang, A. Ardell, S. Zhou, Z. Liu, and L. Chen, Acta Mater. 52, 2837 (2004).

28. A. Dinsdale, Calphad 15, 317 (1991).

29. Metals data book, Japan Institute of Metals, Maruzen, Tokyo, Japan (2004).

30. P. J. Othen, M. L. Jenkins, and G. D. W. Smith, Phil Mag A 70, 1 (1994).

31. R. Becker, Anna. Phys. 424, 128 (1938). 